\title{
Thirty years after the bupivacaine controversy: what have we learned?
}

\author{
James Shannon, FCARCSI · Joanne Douglas, MD
}

Published online: 27 January 2010

(C) Canadian Anesthesiologists' Society 2010

In October 1979, Albright wrote an editorial warning of the risks of cardiac toxicity due to bupivacaine and etidocaine. ${ }^{1}$ The editorial followed a report by Prentiss concerning a cardiac arrest in a patient receiving caudal anesthesia with etidocaine. ${ }^{2}$ In that case, cardiovascular collapse followed the rapid onset of convulsions after possible intravascular (iv) injection of etidocaine. To Albright's knowledge, this was the sixth anecdotal case of sudden cardiovascular collapse following presumed $i v$ injection of bupivacaine $(0.5 \%$ or $0.75 \%)$ or etidocaine. He suggested that inadvertent $i v$ administration of clinical doses of etidocaine and bupivacaine, in comparison with other local anesthetics (LAs), could result in almost simultaneous central nervous system and cardiovascular system toxicity without preceding hypoxia. Albright also noted that resuscitation was difficult, prolonged, and, in most situations, the outcome was poor. ${ }^{1}$

Subsequently, many additional cases of cardiovascular collapse were reported to the Federal Drug Administration (FDA) in the United States. ${ }^{3}$ A worrying number of these cases occurred in young previously healthy women who received epidural analgesia/anesthesia for childbirth. ${ }^{3} \mathrm{Al}-$ bright's editorial and the reports to the FDA prompted a letter to anesthesiologists in 1984 stating that $0.75 \%$ bupivacaine was no longer recommended for obstetric anesthesia because of the risk of cardiac arrest. ${ }^{3}$ Following this letter, the manufacturers of bupivacaine sent similar correspondence to Canadian anesthesiologists that caused

\footnotetext{
J. Shannon, FCARCSI $\cdot$ J. Douglas, MD $(\bowtie)$

Department of Anesthesia, British Columbia's Women's

Hospital and Health Centre and University of British Columbia,

Room 1Q72, 4500 Oak Street, Vancouver,

BC V6H 3N1, Canada

e-mail: jdouglas@cw.bc.ca
}

considerable controversy and debate. ${ }^{3}$ In other countries, caution was urged in using $0.75 \%$ bupivacaine, although many anesthesiologists felt that the problem of cardiotoxicity was confined to the United States. ${ }^{4,5}$

In this issue of the Journal, Dillane and Finucane ${ }^{6}$ review the systemic toxicity of LAs, describing the mechanisms underlying central nervous system and cardiovascular toxicity associated with elevated plasma levels of LAs. They point out that systemic toxicity of LAs frequently results from inadvertent $i v$ injection or from rapid absorption of the LA from the injection site with subsequent high plasma levels, and they suggest that there are benefits from the use of ultrasound during the performance of regional anesthesia. ${ }^{6}$ Ultrasound not only facilitates the identification of anatomical structures, hence avoiding $i v$ injection, but also, it can facilitate more targeted administration of smaller volumes of LAs, thereby increasing the safety of regional anesthesia.

What can we learn from this review article and other similar articles? First, the authors reiterate the history and the known facts regarding this potentially fatal complication. Second, by so doing, they encourage practicing anesthesiologists to examine their own practices and to consider whether LA administration is safe in light of the evidence presented. Although Albright ${ }^{1}$ and others implicated bupivacaine's greater toxicity as the direct cause of cardiac arrest, this assessment was not universally accepted. As most drugs that are used for anesthesia are toxic if used in the wrong dose or wrong space, some anesthesiologists urged caution in blaming bupivacaine. These anesthesiologists questioned the anesthetic technique, as several of the obstetric cases presented to the FDA did not reflect what was considered good anesthetic practice at the time..$^{7-9}$ In some of these cases, a large volume of a high concentration of LA was injected through the epidural 
needle, or there was a lack of preparedness for an adverse reaction (e.g., no intravenous), or there was delay in instituting appropriate resuscitation. Considerable debate was generated between clinicians who wanted to blame the drug and clinicians who wanted to blame the anesthetic technique.

Albright recommended animal studies in order to define the relationship between central nervous system and cardiac toxicity and determine the preferred method of treating cardiotoxicity. ${ }^{1}$ Numerous animal studies confirmed the greater toxicity of the highly-lipophilic LAs, which led to the development of the single stereoisomer LAs, levobupivacaine and ropivacaine, that were recommended by many practitioners as a substitute for bupivacaine. $^{6}$ In spite of this recommendation, bupivacaine is still widely used, ${ }^{6}$ but the $0.75 \%$ formulation of bupivacaine is not advised for obstetric patients.

Also, changes to anesthetic practice followed Albright's editorial, in particular, the dispensation of a test dose prior to administration of a large volume of a concentrated LA. The initial recommendation was that administering an epinephrine-containing test dose could detect an $i v$ needle or catheter. ${ }^{10}$ While true in the non-pregnant population, the use of an epinephrine-containing test dose in the parturient has not been universally accepted due to a high false positive rate ${ }^{11}$ and the potential to decrease uteroplacental blood flow and prolong labour due to decreased uterine activity.

Rather than administering a test dose, some authors suggest that aspiration and careful observation are sufficient to detect an $i v$ catheter in the labouring parturient. ${ }^{12}$ However, not all authors agree. Although many anesthesiologists say they use a test dose as part of labour epidural analgesia, a survey from the UK found that this was not always true. ${ }^{13}$ Several authors have recommended that "every dose (of LA) should be a test dose"; in other words, whenever an epidural is being administered to a parturient, an incremental injection of the LA should be combined with close observation. ${ }^{14}$ Many anesthesiologists follow this practice when administering LA, even those who use a test dose containing epinephrine.

Dillane and Finucane point out several aspects of maternal physiology that alter the pharmacokinetics of LA and increase the parturient's risk of toxicity. ${ }^{6}$ Initially, it was suggested that ovine and possibly human pregnancy per se increased the propensity for cardiotoxicity with bupivacaine, ${ }^{15}$ but this was not confirmed in a larger study comparing bupivacaine with ropivacaine. ${ }^{16}$ In this latter study, more ropivacaine was required to induce cardiovascular collapse, ${ }^{16}$ leading some anesthesiologists to believe that ropivacaine was safer. However, others feel the more likely reason was the difference in effectiveness of the two drugs at equal concentrations. ${ }^{6}$
Dillane and Finucane have outlined a suggested protocol for treatment of LA toxicity; ${ }^{6}$ however, there is one additional essential step for the parturient who develops LA toxicity. Work in a canine model showed that resuscitation from bupivacaine-induced cardiotoxicity was more difficult during partial inferior vena cava occlusion. ${ }^{17}$ Therefore, uterine displacement is essential during resuscitation from LA toxicity in advanced pregnancy, and successful maternal resuscitation may require early delivery of the fetus, as uterine displacement is not always effective.

Intravenous lipid emulsion appears to be a useful tool for treatment of LA-induced cardiovascular collapse, but the exact mechanism is unclear. ${ }^{6}$ Another potential treatment is the use of pegylated anionic liposomes to reduce free drug concentration of LA. ${ }^{18}$ These liposomes demonstrate high-affinity binding to LA due to their bilayer structure, their surface charge, and the lipophilic nature of LA.

The incidence of systemic LA toxicity has decreased since Albright's editorial ${ }^{1}$, due in part to changes to anesthetic practice and the development of novel stereoselective LAs. This decrease has been particularly notable in the area of obstetric anesthesia where the case fatality rate for regional anesthesia for Cesarean delivery in the United States decreased from 8.6 per million in 1979-1984 to 1.9 per million in $1985-1990 .{ }^{19}$ Changes in anesthetic practice, such as careful aspiration prior to injecting LA, incremental injection of LA through a catheter, use of a test dose, close observation of the parturient when injecting LA, and use of more dilute LAs for labour analgesia, have been primarily responsible for this dramatic improvement in maternal mortality related to regional anesthesia. In their review, Dillane and Finucane remind us that it is not only the drugs that we use that are important but also the manner in which they are used.

\section{Controverse au sujet de la bupivacaïne, trente ans après: qu'avons-nous appris?}

En octobre 1979, Albright rédigeait un éditorial nous mettant en garde contre les risques de toxicité cardiaque provoquée par la bupivacaïne et l'étidocaïne. ${ }^{1}$ Cet éditorial faisait suite à un compte-rendu de Prentiss qui décrivait l'arrêt cardiaque d'un patient ayant reçu une anesthésie caudale à base d'étidocaïne. ${ }^{2}$ Dans ce cas précis, la survenue rapide de convulsions a été suivie par un collapsus cardiovasculaire après une possible injection intravasculaire (IV) d'étidocaïne. Albright déclarait alors qu'à sa connaissance, il s'agissait du sixième cas anecdotique de collapsus cardiovasculaire subit après une 
injection présumée en IV de bupivacaïne $(0,5 \%$ ou $0,75 \%)$ ou d'étidocaïne. Son hypothèse était que l'administration IV par inadvertance de doses cliniques d'étidocaïne et de bupivacaïne, au contraire d'autres anesthésiques locaux (AL), pouvait provoquer une toxicité quasi simultanée du système nerveux central et du système cardiovasculaire et ce, sans hypoxie préliminaire. Albright notait également que la réanimation était difficile, prolongée et, dans la plupart des cas, avait un mauvais pronostic. ${ }^{1}$

Par la suite, plusieurs autres cas de collapsus cardiovasculaire se sont ajoutés à la liste des cas rapportés à la FDA (Food and Drug Administration) aux États-Unis. ${ }^{3}$ Une proportion inquiétante de ces cas étaient survenus chez des patientes jeunes et auparavant en bonne santé qui avaient reçu une analgésie/anesthésie péridurale pour le travail obstétrical. ${ }^{3}$ À la suite de l'éditorial d'Albright et du nombre inquiétant de cas signalés à la FDA, une lettre avait été envoyée aux anesthésiologistes en 1984 stipulant que de la bupivacaïne à $0,75 \%$ n'était plus recommandée pour l'anesthésie obstétricale à cause du risque d'arrêt cardiaque. ${ }^{3}$ Cet envoi a été suivi par une missive similaire des fabricants de bupivacaïne adressée aux anesthésiologistes canadiens, causant une véritable controverse et des débats animés. ${ }^{3}$ Dans les autres pays, la prudence a été recommandée lors de l'utilisation de bupivacaïne 0,75\%-mais nombreux sont ceux qui pensaient que le problème de la cardiotoxicité se limitait aux États-Unis! $!^{4,5}$

Dans ce numéro du Journal, Dillane et Finucane ${ }^{6}$ passent en revue la toxicité systémique des anesthésiques locaux et décrivent les mécanismes qui sous-tendent la toxicité sur les systèmes nerveux central et cardiovasculaire associée aux taux plasmatiques élevés de ces agents. Ils soulignent que la toxicité systémique des anesthésiques locaux est souvent le résultat d'une injection IV commise par inadvertance ou de l'absorption rapide de l'AL depuis le site d'injection suivie de taux plasmatiques élevés. Les auteurs suggèrent que l'utilisation de l'échoguidage peut être avantageuse pendant la réalisation d'une anesthésie régionale. ${ }^{6}$ En effet, non seulement l'échoguidage facilite-t-il l'identification des structures anatomiques, ce qui permet d'éviter une injection $\mathrm{IV}$, mais cette technique peut également favoriser une administration plus ciblée de volumes moindres d'AL, ce qui augmente l'innocuité de l'anesthésie régionale.

Que pouvons-nous apprendre d'un article de synthèse tel que celui présenté ici? En premier lieu, cet article nous présente l'historique de cette complication potentiellement fatale et de ce que nous en savons. Deuxièmement, il encourage l'anesthésiologiste en pratique clinique à examiner sa propre pratique et à évaluer sa sécurité, compte tenu des données probantes présentées. Bien qu'Albright ${ }^{1}$ et d'autres aient retenu la plus grande toxicité de la bupivacaïne comme étant la cause directe de l'arrêt cardiaque, cette hypothèse ne fait pas l'unanimité. La plupart des médicaments utilisés en anesthésie sont toxiques s'ils sont mal dosés ou mal administrés; pour cette raison, certains anesthésiologistes ont appelé à la prudence dans la mise au banc de la bupivacaïne. Ces anesthésiologistes ont remis en question la technique anesthésique utilisée dans ces cas de toxicité, étant donné que plusieurs des cas obstétricaux présentés à la FDA ne reflétaient pas ce qui était alors considéré comme une bonne pratique de l'anesthésie. ${ }^{7-9}$ Dans certains de ces cas, un volume important d'une concentration élevée d'AL a été injecté par l'aiguille péridurale, ou il manquait de préparation en cas d'effet secondaire (par ex., pas d'intraveineuse), ou alors il y a eu un délai prolongé avant de débuter une réanimation adéquate. Le débat a fait rage entre ceux qui voulaient blâmer le médicament et ceux qui voulaient blâmer la technique d'anesthésie.

Selon Albright, des études animales étaient nécessaires afin de définir la relation entre le système nerveux central et la toxicité cardiaque et la meilleure façon de traiter la cardiotoxicité. ${ }^{1}$ Plusieurs études animales ont confirmé la toxicité plus importante des $\mathrm{AL}$ très lipophiles, ce qui a motivé la mise au point d'AL stéréo-isomères uniques, la lévobupivacaïne et la ropivacaïne, que plusieurs recommandent comme substitut à la bupivacaïne. ${ }^{6}$ Malgré cette recommandation, la bupivacaïne est encore couramment

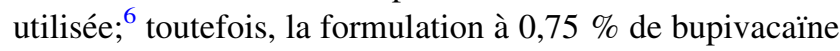
est désormais déconseillée en obstétrique.

Certaines modifications ont également été apportées à la pratique de l'anesthésie à la suite de l'éditorial d'Albright, notamment l'utilisation d'une dose de test avant l'administration d'un volume important d'AL concentré. La recommandation initiale était qu'une dose de test contenant de l'épinéphrine pouvait détecter la position intraveineuse d'une aiguille ou d'un cathéter. ${ }^{10}$ Bien qu'utile dans la population non enceinte, l'utilisation d'une dose de test contenant de l'épinéphrine chez la parturiente n'a pas été unanimement accepté en raison d'un taux élevé de faux positifs, ${ }^{11}$ ainsi que du potentiel de prolongation du travail liée à une activité réduite de l'utérus et une réduction du débit sanguin utéro-placentaire.

Certains auteurs suggèrent que l'aspiration et une observation méticuleuse, plutôt qu'une dose de test, suffisent à détecter un cathéter IV chez la parturiente en travail. $^{12}$ Cependant cet avis ne fait pas non plus l'unanimité et, bien que nombre d'anesthésiologistes déclarent utiliser une dose de test dans le cadre de leur analgésie péridurale pour le travail obstétrical, un sondage réalisé au Royaume-Uni a découvert que ce n'était pas toujours vrai. ${ }^{13}$ Pour nombre d'auteurs, « chaque dose (d'AL) devrait être une dose de test »; en d'autres mots, chaque fois qu'une péridurale est administrée à une parturiente, l'injection progressive de l'AL devrait être combinée à une observation rigoureuse. ${ }^{14}$ Cette pratique 
est suivie par de nombreux anesthésiologistes lorsqu'ils donnent un AL, même par ceux qui utilisent une dose de test contenant de l'épinéphrine.

Dillane et Finucane soulignent plusieurs aspects de la physiologie maternelle qui modifient la pharmacocinétique des AL, augmentant ainsi le risque de toxicité chez la parturiente. ${ }^{6}$ À l'origine, il a été suggéré que la grossesse ovine, et possiblement humaine, augmentait en soi la propension à une cardiotoxicité de la bupivacaïne; ${ }^{15}$ toutefois, cette hypothèse n'a pas été confirmée dans une étude plus importante comparant la bupivacaïne à la ropivacaïne. ${ }^{16}$ Dans cette seconde étude, il a fallu utiliser des doses plus importantes de ropivacaïne pour induire un collapsus cardiovasculaire, ${ }^{16}$ ce qui a incité certains à croire que la ropivacaïne était plus sécuritaire. Cependant, d'autres estiment que la différence d'efficacité entre les deux médicaments à concentrations égales était la raison la plus probable. ${ }^{6}$

Dillane et Finucane ont esquissé un protocole pour le traitement de la toxicité des AL. ${ }^{6}$ Mais pour la parturiente qui manifeste une toxicité à un $\mathrm{AL}$, il y a une étape supplémentaire cruciale. Des travaux réalisés sur des modèles canins ont montré que la réanimation suivant une cardiotoxicité provoquée par la bupivacaïne était plus difficile lors d'une occlusion partielle de la veine cave inférieure. ${ }^{17}$ Pour cette raison, le déplacement de l'utérus est indispensable pendant la réanimation à la suite d'une toxicité par AL. Toutefois, le déplacement de l'utérus n'étant pas toujours efficace, il peut s'avérer nécessaire d'accoucher précocement le fœtus pour parvenir à réanimer la mère.

Un outil utile pour le traitement du collapsus cardiovasculaire dû aux AL semble être l'émulsion lipidique intraveineuse, mais le mécanisme exact demeure obscur. $^{6}$ Un autre traitement possible est l'utilisation de liposomes anioniques pégylés afin de réduire la concentration médicamenteuse libre de l'AL. ${ }^{18} \mathrm{La}$ liaison de ces liposomes avec les AL démontre une affinité élevée en raison de leur structure bicouche, de leur charge de surface et de la nature lipophile des AL.

Depuis la publication de l'éditorial d'Albright, ${ }^{1}$ l'incidence de toxicité systémique aux AL a diminué. Ceci s'explique en partie par les changements apportés à la pratique de l'anesthésie mais également par la mise au point d'AL stéréo-sélectifs innovants. Cette réduction est particulièrement prononcée dans le domaine de l'anesthésie obstétricale, où le taux de létalité de l'anesthésie régionale lors d'accouchements par césarienne aux États-Unis a considérablement diminué, passant de 8,6 par million dans la période 1979-1984 à 1,9 par million dans la période 1985-1990. ${ }^{19}$ Les changements apportés à la pratique de l'anesthésie, comme par exemple l'aspiration méticuleuse avant l'injection d'un AL, l'injection progressive d'AL dans un cathéter, l'utilisation d'une dose de test, l'observation minutieuse de la parturiente pendant l'injection de l'AL et l'utilisation d'AL plus dilués pour l'analgésie pour le travail obstétrical, sont les principaux responsables de cette amélioration marquée de la mortalité maternelle liée à l'anesthésie régionale. Le compte-rendu de Dillane et Finucane nous rappelle que ce ne sont pas seulement les médicaments que nous utilisons qui sont importants, mais aussi la façon dont nous les utilisons.

Competing interests None declared.

\section{References}

1. Albright GA. Cardiac arrest following regional anesthesia with etidocaine or bupivacaine. Anesthesiology 1979; 51: 285-7.

2. Prentiss JE. Cardiac arrest following caudal anesthesia. Anesthesiology 1979; 50: 51-3.

3. Writer WD, Davies JM, Strunin L. Trial by media: the bupivacaine story. Can Anaesth Soc J 1984; 31: 1-4.

4. Beeby DG, Jenkins JG. The bupivacaine controversy (Letter). Anaesthesia 1984; 39: 841.

5. Scott DB. Toxicity caused by local anaesthetic agents. Br J Anaesth 1984; 56: 435-6.

6. Dillane D, Finucane BT. Local anesthetic systemic toxicity. Can J Anesth 2010; 57: this issue.

7. Batra MS, Bridenbaugh LD, Caldwell CB, et al. Bupivacaine cardiotoxicity in a pregnant patient with mitral valve prolapse: an example of improperly administered epidural block (Letter). Anesthesiology 1984; 60: 170-1.

8. Knapp RM. Bupivacaine cardiotoxicity may be more related to technique than to the drug (Letter). Anesthesiology 1984; 60: 257-8.

9. Gambling DR, McMorland GH. "Single-shot" epidural anaesthetics (Letter). Can J Anaesh 1988; 35: 325-7.

10. Moore DC, Batra MS. The components of an effective test dose prior to epidural block. Anesthesiology 1981; 55: 693-6.

11. Guay J. The epidural test dose: a review. Anesth Analg 2006; 102: $921-9$

12. Norris MC, Ferrenbach D, Dalman H, et al. Does epinephrine improve the diagnostic accuracy of aspiration during labor epidural analgesia? Anesth Analg 1999; 88: 1073-6.

13. Gardner IC, Kinsella SM. Obstetric epidural test doses: a survey of UK practice. Int J Obstet Anesth 2005; 14: 96-103.

14. Van Zundert A, Vaes L, Soetens $M$, et al. Every dose given in epidural analgesia for vaginal delivery can be a test dose. Anesthesiology 1987; 67: 436-40.

15. Morishima HO, Pederson H, Finster M, et al. Bupivacaine toxicity in pregnant and nonpregnant ewes. Anesthesiology 1985; 63: 134-9.

16. Santos AC, Arthur GR, Wlody D, De Armas P, Morishima HO, Finster $M$. Comparative systemic toxicity of ropivacaine and bupivacaine in nonpregnant and pregnant ewes. Anesthesiology 1995; 82: 734-40.

17. Kasten $G W$, Martin ST. Resuscitation from bupivacaine-induced cardiovascular toxicity during partial inferior vena cava occlusion. Anesth Analg 1986; 65: 341-4.

18. Howell BA, Chauhan A. Bupivacaine binding to pegylated liposomes. Anesth Analg 2009; 109: 678-82.

19. Hawkins JL, Koonin LM, Palmer SK, Gibbs CP. Anesthesiarelated deaths during obstetric delivery in the United States, 1979-1990. Anesthesiology 1997; 86: 277-84. 